\title{
Geosimulation and its Application to Urban Growth Modeling
}

Paul M. Torrens (2006). In: Complex Artificial Environments, J. Portugali (Eds),

London: Springer-Verlag, pp. 119-134

\begin{abstract}
Automata-based models have enjoyed widespread application to urban simulation in recent years. Cellular automata (CA) and multi-agent systems (MAS) have been particularly popular. However, CA and MAS are often confused. In many instances, CA are paraphrased as agent-based models and simply re-interpreted as MAS. This is interesting from a geographical standpoint, because the two may be distinguished by their spatial attributes. First, they differ in terms of their mobility: CA cannot „move”, but MAS are mobile entities. Second, in terms of interaction, CA transmit information by diffusion over neighborhoods; MAS transmit information by themselves, moving between locations that can be at any distance from an agent's current position. These different views on the basic geography of the system can have important implications for urban simulations developed using the tools. It may result in different space-time dynamics between model runs and may have important consequences for the use of the models as applied tools. In this chapter, a patently spatial framework for urban simulation with automata Tools is described: Geographic Automata Systems (GAS). The applicability of the GAS approach will be demonstrated with reference to practical implementations, showing how the framework can be used to develop intuitive models of urban dynamics.
\end{abstract}

\section{Introduction}

The practice of model-design, model-building, and the application of models in the geographical sciences is in the midst of a transformation. Recent shifts in the art and activity of spatial simulation may be considered as the end-result of a decade or so of research and development, currently gathering critical momentum. This is manifest, most vividly, in the emergence of a new class of models, and a new generation of applications, an approach that some authors have begun to refer to as geosimulation (Benenson and Torrens 2004a; Benenson and Torrens 2004d).

In this chapter, we will explore the concept of geosimulation, in the context of its use in building urban models. We will introduce a new methodology for constructing geosimulation models, focused on the idea of spatial automata deviceswhat we call Geographic Automata Systems. We will also demonstrate the use of these techniques for urban applications, referring to the development of simulations of urban growth.

In section 2 geosimulation is discussed as a new approach to simulation, which is defined more concretely in section 3. Automata are introduced in section 4 as the favored modeling tool for geosimulation work. In section 5, it is argued that there is strong need for a patently spatial set of geosimulation tools (elsewhere in this volume, Benenson and colleagues describe software for this very purpose). Our work in this area is introduced in section 6, with reference to Geographic Automata Systems. The problem of modeling urban growth—sprawl in particu- 
lar-is discussed in section 7. Naturally, we argue for a geosimulation approach and we describe an urban growth model based on geosimulation ideas, and built as a Geographic Automata System, in section 8. The practical use of this model to explore growth phenomena is described in section 9, before concluding remarks appear in section 10 .

\section{Geosimulation as a new trend in spatial simulation}

There is a distinguished lineage to the development of spatial simulation methodology, and geosimulation represents what we might consider as the new wave of a long line of spatial simulation developments. The idea behind geosimulation is best considered in terms of the distinction between that approach to modeling and what may have come before it. The distinction between older and newer is not discrete; very little is in the world of geography.

More conventional spatial simulation is perhaps aptly considered as dealing with the exchange of entities and activities between relatively coarsely-considered units of space, and describing those exchanges in relatively aggregate terms. That is a naïve characterization, but it is only intended to serve a comparative use. By means of contrast, we can consider newer-style approaches-our notion of geosimulation-as extending, substituting, and supplanting conventional models. The geosimulation approach is more likely to be characteristic of models that handle massive quantities of geographic entities, each represented at an atomic (individual and independent) scale of consideration. Exchanges of and between these entities is mediated by the connections that exist between elementary components of geographical systems, considered dynamically and interactively. Our ability to simulate geographical phenomena has advanced to the point where entity-level behaviors can be translated, directly, into artificial computational environmentscode. That code can be used to generate and play with incredibly life-like geographic systems - spaces, phenomena, entities-in completely artificial simulated environments; in silico, as Steven Levy might refer to them.

Of course, the distinction between possessing the ability to do something, and actually doing it, is rather important in simulation contexts. Spatial models are hungry things and developers must feed them data, methodology, and tools, before they can get them to perform any tricks. We think our Geographic Automata Systems can help.

Abstracting from geography for a moment, geosimulation could be thought of in terms of broader trends in general simulation. We might draw analogies between geosimulation and parallel developments in the social and physical sciences: bottom-up modeling as an alternative or extension of top-down simulation (Epstein, 1999); open and transparent simulation in lieu of black-box modeling (Wiener, 1961); notions of phenomena as complex adaptive systems (Johnson, 2001), etc. The contribution of geographers to these developments is significant. Geographers are building new tools within a larger simulation community (Dibble and Feldman, 2004), some open source in nature (Clarke and Gaydos, 1998), and others to be shared as software (Benenson et al., 2004; Semboloni et al., 2004). 
The contribution of geographer's ideas to a growing debate about real-world systems should not be under-estimated; space is beginning to feature prominently in cross-disciplinary theory-building and testing in this context (Gimblett, 2002). Rather than poaching methodologies, tools, and ideas from other fields; geography is beginning to have a significantly reciprocal influence across a wide range of fields on the outskirts of its interests. To a certain extent, geosimulation is a catalyst for this activity.

\section{Defining geosimulation}

We have devoted lots of dead trees to specifying geosimulation as a modeling approach, and readers that are particularly interested in that material might wish to read some of that work (Benenson and Torrens, 2004a; Benenson and Torrens, 2004c; Torrens, 2004). Put succinctly, geosimulation might be defined with reference to its explicit attention to space and geography, both methodologically and in terms of its intellectual foundations.

First, we can consider issues of representation in geosimulation models. Whereas more conventional spatial modeling handles representation of geographic units in a relatively aggregate fashion, geosimulation-style models are more judicious in their representation of geography. The traditional consideration of average and spatially-modifiable geographical units or (statistically) mean individuals is replaced in geosimulation; units are regarded, instead, as spatially nonmodifiable entities, with individual descriptions and independent functionality. Where aggregates are considered, they are more than likely formulated generatively, built from the bottom up by assembling individual entities for the purposes of accomplishing an aggregate task or amassing an aggregate structure.

Second, the treatment of behavior in geosimulation models is important. Under the geosimulation approach, simulated entities are often individual; likewise, they are commonly independent and autonomous in their behavior. From a synoptic perspective, the behavioral focus is often on disaggregate interactions in a systems setting. The independence is significant; attention turns to the specification of individual-level behaviors, and immediately this casts the developer's attention to issues such as cognition, motivation, mobility, etc. Independence has further implications for considering space-time dynamics; we will discuss this in more detail shortly. The move toward autonomy in behavior simulation is also noteworthy; entity behavior is not necessarily treated as homogenous across the system being considered: coffee in the city, but cocoa in the suburbs. Moreover, and borrowing from complexity studies, collective behavior is often modeled as a by-product of spatial interaction; communities emerge as a function of neighbor interaction, for example, with interaction defined in terms of a range of behaviors from perception to budgeting (Torrens, 2001).

Third, geosimulation is markedly distinct in its treatment of time and dynamics, particularly so when compared against more conventional techniques that are popularly employed in spatial simulation. Under the geosimulation approach, models are commonly designed as event-driven, rather than time-driven. Time in 
such simulations moves within discrete packets of change, based on the internal clocks of simulated components. When put together to form a system, update of these clocks may be synchronous or asynchronous; the methodology is relatively flexible in this regard.

\section{Automata as the favored geosimulation methodology}

Methodologically, geosimulation research and development has been dominated by automata-based approaches to model-building. Cellular automata (CA), and their sibling multi-agent systems (MAS), are particularly popular (O'Sullivan and Torrens, 2000; Torrens, 2002a, 2003, 2004; Torrens and O'Sullivan 2001). We would like to argue that an approach based on spatial-specific processing devices-Geographic Automata-is perhaps more appropriate for geographic research. Let us examine automata before we begin that discussion.

At its heart, an automaton is a processing mechanism (whether tangible, or mathematical). It is a discrete entity endowed with some structural variables (states) and capable of receiving similar information as input from the outside world. A given automaton's states change over time (transition) according to a set of rules; these rules evaluate the internal state of the automaton at a point in time and the information input to the automaton at the same time, to determine the automaton's state in a subsequent point in time (Figure 1a). Changes to automata states operate in a discrete temporal domain. Alan Turing's hypothetical computing device is a classic example.

a)

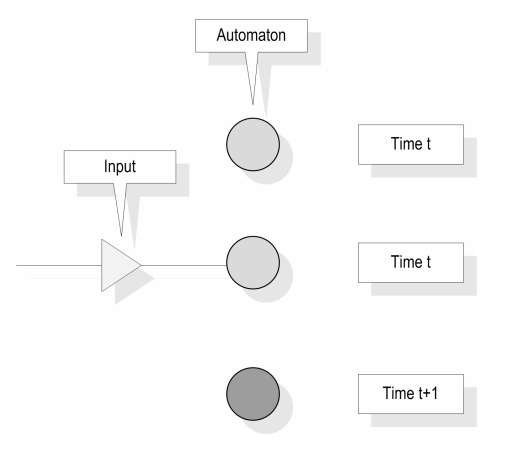

b)

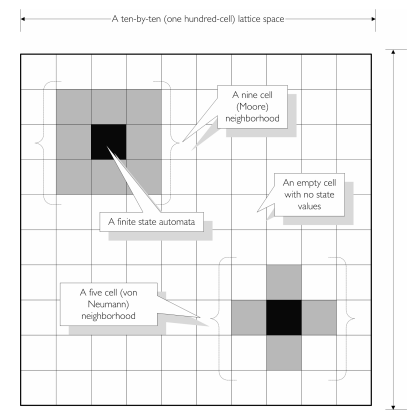

Fig. 1. (a) An automaton changes state (color) between two time steps, based on input. (b) Varying cellular automata neighborhood configurations in a 2D cell-space. 
Cellular automata are an extension of the automata concept, in which the space in which an automaton resides becomes important to the specification. CA are an arrangement of connected individual automata, arranged to form a partitioned space. Think of the pixels on a TV screen and you have the general idea. Each unit in the partition is an automaton as described previously. However, input is now drawn from other automata in a localized area around a given automaton-the neighborhood (Figure 1b).

Multi-agent systems are also automata, although the term has become quite generic in recent years. MAS are generally endowed with some agency; various state variables and transition rules are introduced to lend agent-automata life-like qualities, for example for use in Artificial Intelligence research (Ferber, 1999). In social science, agency is generally expressed with reference to decision-making and choice behavior (Kohler and Gumerman, 2001), cooperation and conflict (Epstein and Axtell, 1996), economic reasoning (Luna and Stefansson, 2000), etc. In other fields, agency is used to mimic insect (Bonabeau et al., 1999) and animal behavior (Meyer and Guillot, 1994), and to specify Internet bots (Leonard, 1997) and Webcrawlers (Pallman, 1999). Agents pop up in all sorts of places, from Lord of the Rings films to Xbox games. In general social science contexts, agents are usually non-spatial in nature. This is not so in geographical contexts, where agency relates quite closely to the mobility of agents in a simulation. This is one of the distinguishing factors between CA and MAS in geographic research; agents may be designed with the ability to move within a simulated space-and carry their state information and rules of interaction with them as they do so. CA, by contrast, are static in their lattice space; they may diffuse information to neighbors, but they cannot alter their position. The distinction is important, at least to geographers. We would like to argue, in fact, that the distinction is so important as to warrant a whole new class of automata-what we call Geographic Automata.

\section{Why we need geographic automata}

Considering my own needs as a model developer, I generally need my model to support some key components of the systems that I wish to simulate. Likely, my needs are quite similar to those of the reader. Of course, I deal mostly with human geographical systems, so my wish-list is understandably biased in that direction. Entities in the model generally need to be distinguishable in terms of the space in which they are situated (or bounded). A flexible expression of the spatial relationships between entities is desirable. Generally, entities in my simulations have some form of mobility, and so I need to be able to track them as they wander around a simulated space, or as their occupation of that space alters. Similarly, I need to be able to describe their behavior, whether spatial or non-spatial.

The trouble with the automata approaches that I outlined in the previous section is that none of them is capable of supporting this sort of functionality in a cohesive manner. CA are handicapped by their inability to simulate true movement. The velocity of information transmission could be approximated in some fashion, but a cell will never be able to uproot and jump around its lattice. MAS can do all of 
this, but much of the current MAS methodology underestimates the importance of space and movement behavior. As desirable as mobility methodology might be to a geographer such as myself, MAS have other shortcomings; agents cannot be fields, for example.

Geography is all about the behavior and distribution of things in space, and different things in different spaces. When we throw time into that soup, we have to consider all of this in the context of space-time dynamics. Why not fall back on our geosimulation approach, infusing spatial properties into the aforementioned automata tools? We might, for example, adopt a fully automata-based view of geographic systems, fabricating systems, from the bottom up and using building blocks fashioned as geographic automata. Relying on notions such as emergence (Johnson, 2001) or network theory (Watts, 2003), we might relate these building blocks to each other as a Geographic Automata System.

\section{Geographic Automata Systems}

Based on our understanding of geographical systems, we can extend the automata idea with space-specific functionality to account for the general needs of geographical modelers. We have actually explored the suitability of the following approach for general urban modeling, at least, and it seems sufficient for most needs that we can consider (Torrens and Benenson, 2005). The usefulness of the approach for modeling urban growth will be demonstrated shortly, but first let us define what we mean by a Geographic Automata System.

A Geographic Automata System retains all of the basic functionality of automata, CA, and MAS:

- States

- State transition rules

- Neighborhoods

To this mixture, we add some peculiarly geographic functionality:

- A typology, describing automata types

- Neighborhood transition rules

- Dynamic location conventions

- Movement rules

The result is a unique class of automata, somewhere beyond CA and MAS, with a dash of Geographic Information Science. The former set of functionalities outlined above now hinge on the latter set-state, state transition rules, and neighborhoods are formulated on the basis of automata type, neighborhood transition rules, location conventions, and movement rules. As ever, all of these components are dynamic with respect to time. In a simulation context, exploration with the Geographic Automata Systems then invokes qualitative and/or quantitative investigation of the influence of these components on system behavior; the ability of the framework to support representation of geographical systems; and specifica- 
tion of the spatio-temporal behavior of geographic entities in an artificial Geographic Automata System simulation environment. The Geographic Automata Systems idea expressed here is a framework for modeling, but it can be used to build models and software for spatial simulation. Thus far we have begun to construct minimal, but wholly sufficient, simulations based on the framework (Benenson and Torrens, 2004b). In addition, Benenson and colleagues have used the framework as the basis for a library of urban simulation tools (Benenson et al., 2004). Before describing one such modeling example in more detail, let us explain the concept in fuller detail.

In the framework, we allow for typologies of Geographic Automata. In our own work thus far, we distinguish between fixed and non-fixed Geographic Automata (GA). Fixed GA are used to model entities that do not change their location over time (although their spatial extent may change in size-shrink or grow, for example-or shape). In an urban context, such entities might be roads, land parcels, or parks. Considering the list of functionality outlined previously, fixed GA may succumb to the influence of state and neighborhood transition rules, but not those of motion. Non-fixed entities are those that have the ability to change their location in space and time. Again, in an urban context, we might think of pedestrian walkers, migrating renters, or subway trains. The full range of rules may be applied to non-fixed GA; location conventions and movement rules are of obvious importance, but neighborhood rules take on a curious form when entities are mobile with respect to other entities, and here the fixture of those GA becomes significant. The movement of a car relative to other cars as opposed to that relative to a traffic light is one example you might consider. The distinction is equally important when employing algorithms, mathematics, or databases in their representation.

GA may also possess state descriptors and state transition rules, as with automata, CA, and MAS. Once again, these may be dynamic with respect to time and space. Their formulation in Geographic Automata Systems differs, however. State transition depends on input from fixed neighborhoods in the context of automata and CA. So, cells appear to magically mutate within a lattice. This is fine for describing phenomena such as local-scale urban decline, but less so for other scenarios-migration is an obvious example. If we consider a model with fixed and nonfixed GA, however, state transition falls under the additional influence of the spatial behavior of other objects in the system, or even within the cell.

Indeed, the addition of functionality to enable, determine, and describe movement of GA within the system opens up all manner of possibilities for developing spatial models, and using them to test theories and simulate phenomena of interest. Movement, and its representation in a simulation context, is a popular research thread in fields peripheral to geography; the video game industry is one example that springs to mind, with an emphasis on movement choreography (Reynolds, 1999). It seems obvious that geographical simulations should accommodate that sort of functionality, as well as adding unique geographical theory and methodologies relating to mobility, search behavior, way-finding, etc. Movement rules have thus been added to the framework. Indeed, as we will demonstrate later, they become a key ingredient of our urban growth models. 
The introduction of functionality to support movement necessitates inclusion of components to track the location of entities and objects in simulated spaces. Considering a typology of geographic entities that is based on fixture, location conventions should be amenable to supporting entities of fixed and non-fixed type, their stable and mobile locations, as well as facilitating evaluation of relationships between the two on the basis of those conventions. In the examples that we have developed thus far, we allow for two varieties of location convention. Direct location is specified rather obviously in terms of the current location of an entity in the system, and this might relate to a coordinate point, additional height information, centroids, a network location, or a set of vectors bounding a polygonal coverage. Location by indirect means is somewhat more complicated, and we have formulated this by means of pointers. An entity has its own location conventions, as well as an additional set of location primitives that express its location relative to other objects or entities. Two brothers might duplicate the same location when at home in their townhouse, but an indirect pointer will be employed to convey the existence of that relationship as they separate in space, for example when they go to different nightclubs on a Friday evening.

This brings us to the issue of neighbors and neighborhood rules. Neighborhoods feature in CA models, as described previously. Similarly, agents in MAS may have neighbors. In CA models, neighborhoods are usually static and symmetrical. They are not really suited to describing dynamic spatial relationships between objects and their variance in space and time. The neighbor concept in MAS is more flexible; flying or swimming Boids may have nearest neighbors for example (Reynolds, 1987). However, geographers' interest in neighborhoods is much broader, encompassing notions such as adjacency, connectivity, and proximity that are not always geometrical in form. A generalized framework for describing such concepts is needed. We allow for such functionality in the Geographic Automata System framework, by separating neighborhoods and neighborhood rules that govern the ways in which those neighborhoods might change in space in time, as Voronoi relations, social networks, leader-follower partnerships, etc. There may be instances in which these are non-spatial, or a mixture of spatial and non-spatial. Consider a household example. Neighbor relations could be expressed in terms of family ties: parent-to-child, sibling-to-sibling. As family members go about their business over the course of a day, the spatial relations between these people will change (or not; siblings may go to the same school), but the family tie remains. If there is a new birth in the family, the family tie will be altered, again nonspatially. As children grow and leave for university, they are de-coupled from the household space and interaction may take on a new form (email, phone). If the family fissions, both spatial and non-spatial relations may change yet again, with parents divorcing and moving apart. The neighbor and neighborhood rules need to be flexible to accommodate these sorts of behaviors.

Next, let us illustrate the use of these components in a unified manner, to build models of urban growth and to run simulations of suburban sprawl. 


\section{An application to simulating sprawl}

We have built the modeling framework to be discussed using a geosimulation approach, and on the basis of a GAS foundation. The model is designed to simulate urban growth. Under simulated conditions, city-systems evolve from initial seed settlement sites, going on to urbanize through compaction, polynucleation, infill, inner-city densification and decline, and peripheral suburban sprawl. We have designed the model as an artificial laboratory to test ideas and hypotheses about sprawl in particular.

A lengthy discussion about sprawl is somewhat beyond the general remit of this text; the reader should consult Torrens \& Alberti (2000) for more substantial treatment of the topic. Put succinctly, sprawl refers to a phenomenon that is particularly prevalent (and popularly studied) in the United States. Sprawl is suburban growth, first and foremost, understood to extend peripherally around cities in swaths of development that are much lower in density than the core area of the city in question; it is also much more scattered in its spatial distribution.

Sprawl has a number of empirical characteristics. It is also manifest with lots of softer, non-quantifiable, characteristics; consult the literature in architecture and urban design for examples (Calthorpe et al., 2001; Duany et al., 2000; Duany et al., 2001; Katz, 1993). A minimal set of descriptors of sprawl might well include measures of density (of population, employment, or some other activity); the functionality of urban space in the city-system; spatial distribution or structure of the urban extent; and dynamic attributes that would allow all of these things to change in space and time. Moreover, these characteristics may be important at varying scales of observation or consideration.

The potential causes of sprawl are numerous and hotly debated (Ewing, 1997; Gordon and Richardson, 1997a). In fact, evaluation of the veracity of debated factors might be one of the goals of a sprawl model. System growth is very important as a sprawl mechanism; it sets the metabolism of the city and sprawling cities are generally either fast-growing in absolute population totals, or fast-growing in the decentralization of that population to the city's periphery (even if it means leaving a donut hole in the center of the city). The distribution of that growth is particularly significant, and this is what distinguishes sprawl from general suburbanization in most instances. The distribution of sprawl is low in density, scattered in nature, rapid in its appetite for land, and is almost always manifest on the periphery of the main urban mass.

Space and geography, then, are absolutely essential to consideration of sprawl. But representation of the spatial components of sprawl necessitates a uniquely spatial modeling approach. Not surprisingly, we would like to argue that sprawl is an ideal test-bed for geosimulation and GAS; similarly, geosimulation and GAS offer much potential for generating and testing ideas relating to sprawl. Let us try to demonstrate that with a modeling example. 


\section{Model description}

We have constructed an urban growth model, formulated on the basis of a Geographic Automata Systems engine, as mentioned, with components of the model formulated in that scheme: a typology of fixed and mobile geographic automata, each described in space and time by means of a set of state variables, state transition rules, geo-referencing conventions, movement rules (if mobile), neighbor conventions, and neighborhood rules.

The typology delineates two types of geographic automata-fixed and mobile. Fixed GA correspond to landscape and infrastructure elements. The simulated space is characterized as a landscape, until developed into urban infrastructure by the other-mobile-type of GA in the model. Mobile GA serve as the agents of change in the simulated system. They are designed to mimic developers and settlers, wandering the landscape with informed behaviors, converting it from nonurban to urban uses, and depositing population as they proceed. In this way, then, fixed GA act as a container for mobile GA; the landscape supports settlement through urbanization.

Fixed GA may also be designated as gateways. Essentially, gateways serve as an entry-point to incoming growth (population) to the simulated system. A priori, certain sites are designated as gateways in the simulated city, corresponding to the initial seed locations from which an urban system evolves. Gateways may also manifest over the course of a simulation run-during run-time-and this is used to denote the emergence of new centers of urbanization within the city-system as it evolves. Whereas the seed gateways are used as a proxy for exogenous inmigration to the city-system, run-time gateways are used to generate endogenous growth (and decline) within the city.

A set of state variables are used to introduce a minimal set of relevant characteristics of the evolving urban system: whether or not a GA is a gateway, the development condition of the simulated landscape, and the state of its settlement. Fixed GA units are endowed with variables to describe whether they are developable or not; this allows for space to be delineated as functional (suitable for urbanization) or not. Similarly, those units are described with an additional variable to indicate whether they have been developed. The end result of urbanization in the model is settlement of a fixed GA with some volume of population; a population count variable is thus introduced to denote the number of people residing on a given fixed GA unit. Because fixed GA are equal in size in the simulation, this may be interpreted as a population density state.

Of course, all of these variables are dynamic with respect to space and time. Change takes place in the model through general state transition rules, as well as the movement activity of mobile GA units. One of the interesting features of employing a GAS-based approach to modeling urban growth is that much of the state transition functionality that appears in traditional CA-style urban growth models (Clarke and Gaydos, 1998; Engelen et al., 1995; White and Engelen, 2000; Xie, 1994; Yeh and Li, 2000) can actually be handled through movement rules, thus avoiding the sort of methodology that would have cell states mutate magically within a lattice, rather than initiating as the result of agent-based activity within 
them. Consequently, a handful of general state transition rules are employed. A dispersal function is used, to distribute population very locally between fixed developed GA units, within a small neighborhood radius. This neighborhood is specified in an eight-cell Moore configuration and the dispersal works by diffusing a percentage of population within that neighborhood to a target cell. This dispersal actually works in two directions: growth is diffused between neighbors, but so too is decline. If the average population in the neighborhood is below the value in a target cell, the total in the reference cell will decline accordingly. In this way, then, phenomena such as urban blight and gentrification are handled, albeit in a proxy manner.

A number of georeferencing conventions are used in the model, to situate important features in the model, such as seed gateways, and to track the movement of mobile GA as they propagate through the system. Georeferencing is performed by direct and indirect means. The actual coordinates of modeled entities within the simulated space are noted by fixed means, as $(\mathrm{x}, \mathrm{y})$ coordinates on a Cartesian plane with origin in the centroid of the simulated space. If a GA is fixed, these coordinates will not change; if it is mobile, the coordinates will be updated to reflect shifts in their position. Indirect georeferencing is employed with respect to mobile GA and the seed gateways from which they originate. As a mobile GA moves through the simulated landscape, its direct location will change, but it retains a pointer to the seed gateway from which it originated in the system. This designation remains with the GA as long as it is present within the system.

Movement rules constitute the real work-horse of the model. The rules are designed to mimic proposed drivers of sprawl, as discussed in the literature. Specifically, we use the geographic factors understood to be responsible for sprawl in American cities as inspiration for the formulation of the movement regimes. Thus, we have specified a variety of forms of movement for the modeled developersettler GA. Growth enters the system either from exogenous or endogenous sources, and that growth is distributed spatially over the urbanizing landscape using mobile GA (Figure 2).

Compact development regimes are mimicked by immediate and nearby movement rules that see mobile GA develop fixed GA in small eight-cell and 24-cell neighborhoods of influence. The compact rules correspond to the sorts of development that might take place early in the evolution of a city-system, when space is considered with relatively less premium than may be the case at a later stage in the city's growth. The compact rules also mimic conventional New Urbanist ideas about denser forms of development (Katz, 1993). The compact movement rules lead to relatively small clusters of dense settlement. 


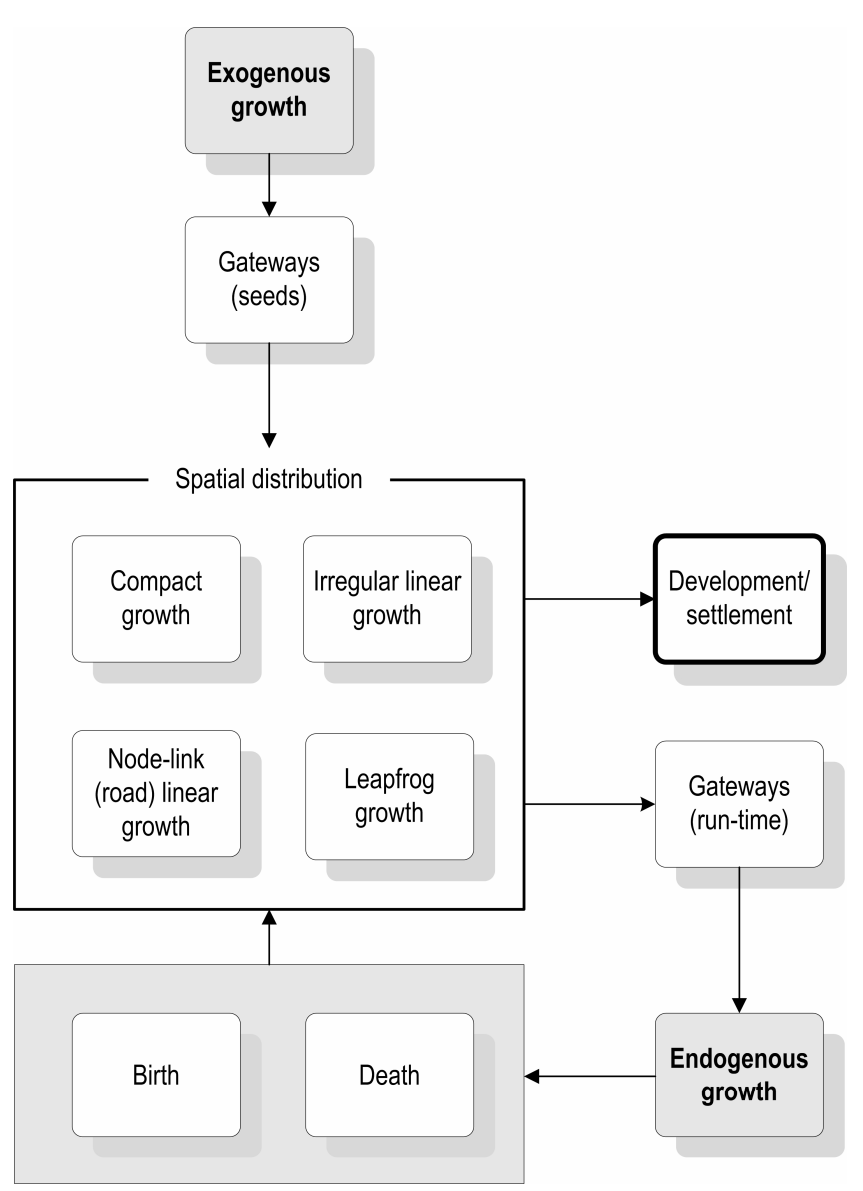

Fig. 2. A conceptual diagram of the urban growth model engine.

More linear forms of development, such as that which might flourish around transport corridors or flanking arterial highways, are modeled using two other movement rules: an irregular and road-like movement regime. The irregular function is used to simulate the sorts of development features that might occur when development is constrained to an irregular linear process due to natural or political boundaries. This rule leads to a dendritic form of development, much like that generated by diffusion-limited aggregation (Batty et al., 1989) or random walks (Batty and Longley, 1994). The road-like movement rule is used to grow roads in a simulation. Rather than having roads pop-up out of the ether, the rule is formulated in a chained node and link manner, whereby a developing GA moves over the landscape, laying down nodes to be connected; these nodes are then linked by linear strips of development, thereby mimicking road-like urbanization. This is particularly useful in simulating ribbon-sprawl, i.e., sprawl that tends to feature in linear swaths buffering major roads on the periphery of cities. 
Fragmentation is a major feature of conventional suburbanization; as mentioned previously the notion of scatter is crucial to sprawl. Scattered development on the periphery of cities, at lower-than-average densities of settlement, is now commonplace in many American cities. It is largely a by-product of speculative land development (Bahl, 1968), often in areas that were previously devoted to agricultural use. In a sense, the formation of edge cities (Garreau, 1992) is also a process of scattering, albeit on a larger scale of consideration. Scattered development is mimicked by means of a leapfrog movement rule, whereby a mobile developer GA can skip ahead of the main urban mass and settle sites outside of that periphery.

The movement rules may also be combined, so that a leapfrog movement may be followed by a road-like movement or a compact movement, or other such combinations.

Thus far, we have covered specification of the model as a Geographic Automata System, referring to the typology of entities in the model, the set of state variables used, state transition rules, georeferencing conventions, and movement rules. The final set of components that we need to describe relate to spatial relationships in the model: neighborhoods and neighborhood rules. As mentioned with respect to the compact movement rules, variable neighborhoods are employed in the model and these allow for different areas of influence for development and settlement to be introduced. In addition, action-at-a-distance is also supported, as in the case of the road-like and leapfrog movement rules. In this way, then, the influence of a given GA may extend beyond the neighborhood filter.

Neighborhood rules are employed in a rather simple fashion. The actual shape of a neighborhood does not vary as the simulation evolves, mobile GA just choose to employ varying sizes of neighborhood, and this choice is tied quite simply to the movement rule that they are ordered to employ in a given place at a given time.

This brings us to broader issues of time and dynamics, and their use in the model. Time is discrete in the model; it moves in packets or bundles of change. These bundles are characterized as a simulated year in the simulation we will present shortly. In this sense, time is event-driven. Each packet of change (a year) may involve hundreds of individual transitions and movements, and these movements will proceed based on their own internal clocks. A road-like movement rule will take up many cycles of the CPU's clock, whereas the in-migration of growth to a gateway may take up only a handful of cycles. The volume of activity that occupies a single temporal packet will understandably grow as the simulated city fills with more and more people-more and more agents of change. But, by encapsulating these dynamics within events time will appear to flow quite organically within the simulation. There is also some theoretical justification to this approach to dynamics in the model. Different processes have different cycles and we wished to accommodate that functionality. The mechanisms designed to diffuse growth and decline between fixed GA are slower (in event time) than those employed by movement rules; the lifecycle of development and settlement events is shorter than that of neighborhood transition through gentrification and decline.

Finally, the model works in a constrained fashion. The state variables for $d e-$ velopable or not-developable conditions allow for the simulated space to be de- 
vised as functional (open to development) or non-functional (closed to development, for example, if the area is a large water mass). Automata models are known to be very sensitive to initial parameterization, and urban growth $\mathrm{CA}$ are quite sensitive to the specification of seed sites. Seed gateways are thus introduced with some a priori understanding of where the model developer would like the citysystem to start growing, although other rival sites may emerge over the course of a simulation run. The general metabolism of a simulation is constrained by use of these seed gateways; at each time-step in a simulation run, a volume of growth is metered to the simulation. Although the actual spatial distribution of that growth is left to the model, the general rate of exogenously-derived growth can be constrained. Similarly, the rate of endogenous growth can be constrained. In some simulations, we have tailored the seed conditions and growth rates to known conditions and are able to generate realistic urban evolution and patterns of growth for real-world city-systems (Torrens, 2002b); moreover, quantitative and structural measurements confirm that the simulated conditions match those present on the ground, although that discussion is beyond the main thread of this chapter.

\section{Simulating urban growth}

We have just described how the model is formulated as a Geographic Automata System, and the functionality that framework affords is particularly useful in modeling urban growth. We will now demonstrate how the model can be used to build realistic simulations of urban growth regimes in artificial cities. These simulations allow for various ideas about the factors responsible for sprawl to be tested in an artificial and controlled computational environment. In particular, we can test the ways in which a city-system might evolve by conventional means (sprawl, in this case), and by smart growth mechanisms in which growth is managed in some sustainable form. This actually echoes a hot debate in the literature at the moment, pertaining to questions of whether sprawl or smart growth is desirable, feasible, cost-effective, and socially just (see Gordon and Richardson, 1997b). The purpose of this chapter is to introduce the idea of geosimulation, Geographic Automata Systems as a framework for geosimulation, and to demonstrate how they might be used to build urban growth models. The simulation experiments described here were designed to test specific ideas about conventional urban growth in American cities, and much of that discussion falls outside of the relevance of this chapter. Nonetheless, some brief description of the simulations may serve to emphasize the points that we are trying to make in this discussion. We will introduce two simulations based on the model described in the last section. One relates to sprawl; the other relates to smart growth.

The sprawl simulation is designed to mimic the general evolution of a contemporary city-system in the United States. Five initial gateways seeds are introduced into the model, with differing growth rates. One seed, in the center of the simulated space, is chosen to dominate a priori, and the assignment of growth to it is established to reflect this. $75 \%$ of the way through a simulation run, the supply of external growth to all sites, save this dominant city, is halted and growth in those 
areas proceeds by endogenous means alone. (Growth rates are thus treated heterogeneously across the city-system.) Theoretical justification for this is as follows. The dominant city is afforded a historical advantage from the outset of the simulation; it was an initial settlement site and has inertia, geographically, and path dependence in terms of system dynamics. As the city-system evolves, that site flourishes and gains a competitive advantage that $75 \%$ of the way through the simulation (when its urban extent reaches the hinterlands of competing sites) begins to draw growth away from its competitors. The city-system evolves to a familiar sprawling pattern, with road-influenced fingers of ribbon sprawl and a sea of low-density and fragmented urbanization on the periphery. Urban decline is evident in the core of the city (Figure 3 ).

a)

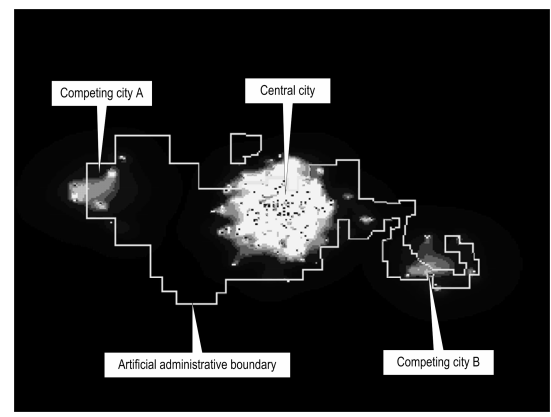

b)

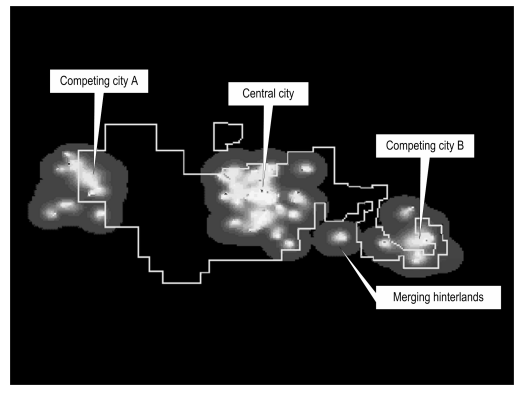

Fig. 3. The results of a sprawl-based (a) and a smart growth (b) simulation run (darker areas refer to low population density; lighter areas house high densities of population).

A second simulation is formulated on a smart growth regime, whereby development is encouraged in smaller compact clusters. A dominant city is established a priori, as before. However, initial peripheral sites gain a competitive advantage outside of the main urban mass, and instead of sprawling become relatively dense in their own right. When the supply of exogenous growth is cut, these settlements actually survive. The result is a polycentric spatial structure to the city-system. Sprawl still predominates on the periphery, but it is bound within edge cities that constitute well-established polycentric cores.

These simulations demonstrate the suitability of the geosimulation approach and the Geographic Automata Systems framework. In related work, we have also begun to use this model to explore ideas about the dynamic formation of sprawl as a generative and inherently spatial process. We have also considered the phenomenon of sprawl at a more micro-scale, and have built a GAS-based model of community dynamics within one single GA "cell" of these simulations (Torrens, 2001), looking at issues of residential mobility, community demographics, and socio-spatial segregation. 


\section{Conclusions}

This chapter has introduced a few new research ideas in the field of urban simulation, hopefully not too generally for the reader to grasp fully. We have proposed geosimulation as a new and particularly useful approach to spatial simulation. Work in the area of geosimulation and urban analysis is really beginning to gather steam; Itzhak Benenson and I have edited a journal's double special issue devoted to the topic (Benenson and Torrens, 2004d). Much of the work discussed in those pages relies on automata tools. In many instances, geosimulation work might benefit from more explicitly spatial simulation tools; the need in geography work is obvious.

We have developed a new framework for geosimulation modeling, focused on automata tools, but with patently spatial (and Geographic Information Science) appeal and functionality-Geographic Automata Systems. We believe the framework to be very useful for modeling urban systems.

One such example is demonstrated here, focusing on urban growth modeling and simulation of scenarios relating to suburban sprawl in American cities. We hope that the framework will be found to be more extensible across a range of geographic examples, relating urban analysis and other areas of geography.

\section{References}

Bahl, R.W. (1968). A land speculation model: the role of the property tax as a constraint to urban sprawl. Journal of Regional Science 8 (2):199-208.

Batty, M., and Longley, P. (1994). Fractal Cities. London: Academic Press.

Batty, M., Longley, P.A. and Fotheringham, A.S. (1989). Urban growth and form: scaling, fractal geometry, and diffusion-limited-aggregation. Environment and Planning $A$ 21:1447-1472.

Benenson, I., and Torrens, P.M. (2004a). Geosimulation: Automata-Based Modeling of Urban Phenomena. London: John Wiley \& Sons.

Benenson, I. (2004b). A minimal prototype for integrating GIS and geographic simulation through Geographic Automata Systems. In GeoDynamics, edited by P. Atkinson, G. Foody, S. Darby and F. Wu. Florida: CRC Press.

Benenson, I., Aronovich, S., and Noam, S. (2004). Let's talk objects. Computers, Environment and Urban Systems, 29 (4): 425-471.

Benenson, I., and Torrens, P.M. (2004c). Geosimulation: object-based modeling of urban phenomena. Computers, Environment and Urban Systems 28 (1/2):1-8.

Benenson, I. \& Torrens, P. M. (2004d). Special Issue: Geosimulation: object-based modeling of urban phenomena. Computers, Environment and Urban Systems 28:1-8.

Bonabeau, E., Dorigo, M., and Theraulaz, G. (1999). Swarm Intelligence: From Natural to Artificial Systems, Santa Fe Institue Studies in the Sciences of Complexity. New York: Oxford University Press.

Calthorpe, P., Fulton, W. and Fishman, R. (2001). The Regional City: Planning for the End of Sprawl. Washington, D.C.: Island Press. 
Clarke, K.C., and Gaydos, L. (1998). Loose coupling a cellular automaton model and GIS: long-term growth prediction for San Francisco and Washington/Baltimore. International Journal of Geographical Information Science 12 (7):699-714.

Dibble, C., and Feldman, P.G. (2004). The GeoGraph 3D Computational Laboratory: Network and Terrain Landscapes for RePast. Journal of Artificial Societies and Social Simulation 7 (1).

Duany, A., Plater-Zyberk, E. and Speck, J. (2000). Suburban Nation: The Rise of Sprawl and the Decline of the American Dream. New York: North Point Press.

Duany, A., Speck, J. and Plater-Zyberk, E. (2001). Smart Growth: New Urbanism in American Communities. New York: McGraw-Hill.

Engelen, G., White, R. Uljee, I. and Drazan, P. (1995). Using cellular automata for integrated modeling of socio-environmental systems. Environmental Monitoring and Assessment 30:203-214.

Epstein, J.M. (1999). Agent-based computational models and generative social science. Complexity 4 (5):41-60.

Epstein, J.M., and Axtell, R. (1996). Growing Artificial Societies from the Bottom Up. Washington D.C.: Brookings Institution.

Ewing, R. (1997). Is Los Angeles-style sprawl desirable? Journal of the American Planning Association 63 (1):107-126.

Ferber, J. (1999). Multi-Agent Systems: An Introduction to Distributed Artificial Intelligence. Harlow (UK): Addison-Wesley.

Garreau, J. (1992). Edge City: Life on the New Frontier. New York: Anchor Books/Doubleday.

Gimblett, H.R. (ed.) (2002). Integrating Geographic Information Systems and Agent-Based Modeling Techniques for Simulating Social and Ecological Processes, Santa Fe Institute Studies in the Sciences of Complexity. Oxford: Oxford University Press.

Gordon, P. and Richardson, H.W. (1997a). Are compact cities a desirable planning goal? Journal of the American Planning Association 63 (1):95-106.

Gordon, P. (1997b). Where's the sprawl? Journal of the American Planning Association 63 (2):275-278.

Johnson, S. (2001). Emergence: The Connected Lives of Ants, Brains, Cities, and Software. London: Allen Lane, The Penguin Press.

Katz, P. (1993). The New Urbanism : Toward an Architecture of Community. New York: McGraw-Hill.

Kohler, T.A., and Gumerman, G. (2001). Dynamics in Human and Primate Societies. New York.: Oxford University Press.

Leonard, A. (1997). Bots: The Origin of a New Species. San Francisco: Hardwired.

Luna, F, and Stefansson, B. (eds.) (2000). Economic Simulation in Swarm: Agent-based Modeling and Object Oriented Programming. Dordrecht: Kluwer.

Meyer, J.A., and Guillot, A. (1994). From SAB90 to SAB94: four years of animat research. In From Animals to Animats 3. Proceedings of the Third International Conference on Simulation of Adaptive Behavior, edited by D. Cliff, P. Husbands, J.-A. Meyer and S. Wilson. Cambridge, MA: The MIT Press, 2-11.

O'Sullivan, D., and Torrens, P.M. (2000). Cellular models of urban systems. In Theoretical and Practical Issues on Cellular Automata, edited by S. Bandini and T. Worsch. London: Springer-Verlag, 108-117.

Pallman, D. (1999). Programming Bots, Spiders, and Intelligent Agents in Microsoft Visual C++, Microsoft Programming Series. Redmond, WA: Microsoft Press. 
Reynolds, C. (1987). Flocks, herds, and schools: A distributed behavioral model. Computer Graphics 21 (4):25-34.

Reynolds, C. (1999). Steering behaviors for autonomous characters. Paper read at Game Developers Conference, at San Jose, CA.

Semboloni, F., Assfalg, J. Armeni, S., Gianassi, R. and Marsoni, F. (2004). CityDev, an interactive multi-agents urban model on the web. Computers, Environment and Urban Systems 28 (1/2):45-64.

Torrens, P.M. (2001). New tools for simulating housing choices. Program on Housing and Urban Policy Conference Paper Series. Berkeley, CA: University of California Institute of Business and Economic Research and Fisher Center for Real Estate and Urban Economics. http://urbanpolicy.berkeley.edu/pdf/torrens.pdf.

Torrens, P.M. (2002a). Cellular automata and multi-agent systems as planning support tools. In Planning Support Systems in Practice, edited by S. Geertman and J. Stillwell. London: Springer-Verlag, 205-222.

Torrens, P.M. (2002b). SprawlSim: modeling sprawling urban growth using automatabased models. In Agent-Based Models of Land-Use/Land-Cover Change, edited by D. C. Parker, T. Berger, S. M. Manson and W. J. McConnell. Louvain-la-Neuve, Belgium: LUCC International Project Office, 69-76.

Torrens, P.M. (2003). Automata-based models of urban systems. In Advanced Spatial Analysis, edited by P. A. Longley and M. Batty. Redlands, CA: ESRI Press, 61-81.

Torrens, P.M. (2004). Geosimulation approaches to traffic modeling. In P. Stopher, K. Button, K. Haynes \& D. Hensher (Eds.). Transport geography and spatial systems. London: Pergamon, pp. 549-565.

Torrens, P.M., and Alberti, M. (2000). Measuring sprawl. CASA Working Paper. London: University College London, Centre for Advanced Spatial Analysis. http://www.casa.ucl.ac.uk/measuring_sprawl.pdf.

Torrens, P.M., and Benenson, I. (2005). Geographic automata systems. International Journal of Geographical Information Science, 19 (4), 385-412.

Torrens, P.M., and O'Sullivan, D. (2001). Cellular automata and urban simulation: where do we go from here? Environment and Planning B 28 (2):163-168.

Watts, D.J. (2003). Six Degrees: The Science of a Connected Age. New York: W.W. Norton \& Company.

White, R., and Engelen, G. (2000). High-resolution integrated modeling of the spatial dynamics of urban and regional systems. Computers, Environment and Urban Systems 24:383-400.

Wiener, N. (1961). Cybernetics: or Control and Communication in the Animal and the Machine. Cambridge, MA: MIT Press.

Xie, Y. (1994). Analytical models and algorithims for cellular urban dynamics. Ph.D., Department of Geography, University of New York at Buffalo, Buffalo, NY.

Yeh, A. Gar-On, and Xia L. (2000). Simulation of compact cities based on the integration of cellular automata and GIS. In Theoretical and Practical Issues on Cellular Automata, edited by S. Bandini and T. Worsch. London: Springer-Verlag, 170-178. 